\title{
Emergency endovascular treatment of cavernous internal carotid artery acute bleeding with flow diverter stent: a single-center experience
}

\author{
Andrea Giorgianni ${ }^{1} \cdot$ Edoardo Agosti $^{2}$ (D) - Alberto Terrana ${ }^{1} \cdot$ Fabio Pozzi $^{2} \cdot$ Giorgio Sileo $^{3} \cdot$ Luca Nativo $^{1}$. \\ Sergio Balbi ${ }^{2}$ - Alessandro Motta ${ }^{4}$ Paolo Castelnuovo ${ }^{3,5} \cdot$ Davide Locatelli $^{2,5}$ - Mario Turri-Zanoni ${ }^{4,5}$
}

Received: 28 May 2020 / Accepted: 30 July 2020 / Published online: 18 August 2020

(C) The Author(s) 2020

\begin{abstract}
Background and objective To describe our single-center experience in the treatment of cavernous internal carotid artery (ICA) acute bleeding with flow diverter stent (FDS), as a single endovascular procedure or combined with an endoscopic endonasal approach. Methods We analyze a case series of 5 patients with cavernous ICA acute bleeding, i.e., 3 iatrogenic, 1 post-traumatic, and 1 erosive neoplastic. After an immediate nasal packing to temporarily bleeding control, patients underwent digital subtraction angiography (DSA) to identify the site of the ICA injury. A concomitant balloon occlusion test (BOT) was performed, to exclude post-occlusive ischemic neurological damage. An FDS was placed with parallel intravenous infusion of abciximab in 3 cases and tirofiban in 2 cases. In two patients, an innovative "sandwich technique" combining the endovascular reconstruction with an extracranial intrasphenoidal cavernous ICA resurfacing with autologous flaps or grafts by endoscopic endonasal approach was performed.

Results No patient had periprocedural ischemic-hemorrhagic complications. All patients had a regular clinical evolution, without general complications or new onset of focal neurological deficits. No further bleeding occurred in 3 patients, while 2 cases experienced a mild rebleeding in a period ranging from 5 to 15 days after the endovascular procedure. In these two cases, we proceeded with an endoscopic endonasal procedure to resurface the exposed ICA wall in the sphenoid sinus.

Conclusions Although the treatment of choice for cavernous ICA acute bleeding remains the occlusion of the injured vessel, in cases of poor hemodynamic compensation at the BTO, the endovascular FDS emergency placement can be effective. A combined endoscopic endonasal technique to support the extracranial side of the vessel using autologous flaps or grafts can be performed to prevent the risk of rebleeding.
\end{abstract}

Keywords Cavernous carotid artery $\cdot$ Acute vascular injury $\cdot$ Flow diverter stent $\cdot$ Skull base surgery $\cdot$ Endoscopic endonasal . Hadad flap

This article is part of the Topical Collection on Vascular Neurosurgery Other

Edoardo Agosti

edoardo_agosti@libero.it

1 Department of Neuroradiology, ASST Sette Laghi, Varese, Italy

2 Division of Neurosurgery, Department of Biotechnology and Life Sciences, University of Insubria, Via Guicciardini, 9, 21100 Varese, Italy

3 Division of Otorhinolaryngology, Department of Biotechnology and Life Sciences, University of Insubria, Varese, Italy

4 Department of Anesthesiology e Resuscitation, ASST Sette Laghi, Varese, Italy

5 Head and Neck Surgery \& Forensic Dissection Research Center (HNS\&FDRc), Department of Biotechnology and Life Sciences, University of Insubria, Varese, Italy

\section{Introduction}

The internal carotid artery (ICA) acute bleeding is one of the most serious neurovascular emergencies that require rapid diagnostic framing and therapeutic targeting. Among the causes of ICA acute injury are traumatic damage, iatrogenic procedure, and neoplastic invasion. Among the carotid segments of the most difficult management in the case of acute rupture is the cavernous tract. In particular, in the case of cavernous ICA acute injury, the control of bleeding can be obtained using an endovascular procedure, endonasal endoscopic approach, or combined techniques [1-6].

Endovascular treatment can be based on either closing the injured vessel or repairing the vascular wall tear. To date, the occlusive technique is preferred to the reconstructive, given its 
best efficacy in bleeding control, both in the short and long term. However, the final choice between these two endovascular procedures depends on whether or not the sacrifice of the injured vessel can be tolerated by the patient in terms of possible post-occlusive neurological deficits. The hemodynamic compensation capacity can be estimated through the determination of the venous phase prominent delay of the injured vessel during balloon occlusion test (BOT) [7]: the greater the delay, the lower the hemodynamic compensation, the major the risk of post-occlusive ischemic damage of the territory downstream of the damaged vessel [8].

Different types of techniques and stents for the management of a cavernous ICA acute injury have been described in the literature. However, a study that offers a comprehensive evaluation of the emergency management of cavernous ICA acute bleeding with flow diverter stent (FDS) endovascular placement is lacking. The aim of this study is to describe our single-center experience in the management and treatment of acute cavernous ICA bleeding with FDS endovascular emergency placement, as an isolated procedure or a combined technique with an endoscopic endonasal trans-sphenoidal cavernous ICA wall reconstruction, providing indications, contraindications, and outcomes.

\section{Materials and methods}

\section{Data collection}

A retrospective review of neuroradiology registry data of patients treated with endovascular FDS placement from 2015 to 2019 was performed. Inclusion criteria were patients with cavernous ICA acute bleeding, with pathological BOT positive for post-occlusive ischemic risk, and treated in an emergency with FDS release. Of these, only patients over the age of 18 and who gave their informed consent were included.

Clinical, pathological, and radiological data were retrieved from the review of medical records and available radiological imaging and prospectively collected in a dedicated database. Clinical data included sex and age of the patient, cavernous ICA injury cause, treatment, complications, and outcome. Radiological data comprehended type of FDS and follow-up radiological investigations, while pathological data included the site and type of the lesion.

\section{Endovascular procedure}

Regardless of cavernous ICA injury etiopathology, each patient underwent a digital subtraction angiography (DSA) to define the location and extension of the vascular wall injury. During the angiographic study, a BOT was performed to calculate the venous phase delay and assess the hemodynamic compensation capacity of the cerebral circulation [7].
Vascular anatomy was also studied, to select the FDS that potentially best suited the individual patient's conformation.

In detail, in all 5 cases, DSA was performed in an emergency regimen, in general anesthesia with a femoral artery access. The BOT was immediately practiced and the blood pressure was maintained in a variable range between 70 and $80 \mathrm{mmHg}$ for diastolic blood pressure and between 110 and $120 \mathrm{mmHg}$ for systolic blood pressure. The parent vessel occlusion test was standardized to a duration of $10 \mathrm{~min}$.

After a multidisciplinary discussion of each case, FDS was placed. During stent deployment, antiplatelet protocol included $8 \mathrm{mg}$ of abciximab (used in 3 cases) or $10 \mathrm{mg}$ of tirofiban (used in 2 cases). A subsequent 12 -h infusion of a saline solution with the same antiplatelet drug $(9 \mathrm{mg}$ in $200 \mathrm{ml}$ of saline solution at $17 \mathrm{ml} / \mathrm{h}$ for abciximab and $10 \mathrm{mg}$ in 100 of saline solution at $11 \mathrm{ml} / \mathrm{h}$ for tirofiban) was performed.

Double antiplatelet therapy with clopidogrel $75 \mathrm{mg}$ plus acetylsalicylic acid (ASA) $100 \mathrm{mg}$ was initiated the day after the procedure and it was maintained for 1 month, while single antiplatelet ASA $100 \mathrm{mg}$ therapy was maintained for other 3 months, for a total of 4 months of antiplatelet therapy.

\section{Endoscopic endonasal procedure}

In two out of 5 cases of rebleeding after endovascular FDS emergency placement, an endoscopic endonasal transsphenoidal procedure was performed to resurface with autologous tissue the cavernous ICA injured wall. After a total spheno-ethmoidectomy, the lateral wall of the sphenoid sinus was inspected with angled scopes (Karl Storz, Tüttlingen, Germany) and the cavernous segment of the ICA was identified. The bony edges around the carotid pseudoaneurysm (PSA) were exposed and denuded from mucosa. The cavernous ICA wall was then covered with an autologous graft (i.e., inferior turbinal mucoperichondrium graft) in one case, and in the other patient, a pedicled nasoseptal Hadad flap was harvested and rotated to resurface the lateral wall of the sphenoid, including the cavernous tract of the ICA (Fig. 1a, b, c). According to this technique, a double reconstruction of the injured ICA wall was performed: an endovascular cavernous ICA endoluminal reconstruction by FDS positioning and an extracranial endonasal reconstruction with autologous flap or graft. We defined this innovative combined endovascularendonasal procedure as "sandwich technique." Nasal packing was placed and then removed after $48 \mathrm{~h}$. A peri-operative third-generation cephalosporin was administered intravenously for 7 days.

\section{Follow-up}

The neuroradiological follow-up was carried out by CTA and DSA, following a standard scheme for each patient. After the FDS release, a DSA control was performed, to verify the FDS 
Fig. 1 Endoscopic endonasal "sandwich technique" with

Hadad flap harvesting (case \#3). a Left sphenoidotomy. $\mathbf{b}$

Harvesting of left nasoseptal flap

(Hadad flap). c Nasoseptal flap placed to cover the left sphenoidotomy. d Endonasal endoscopic image at 22 months, showing complete healing of the nasoseptal flap in the sphenoid as well as the nasal septum donor site
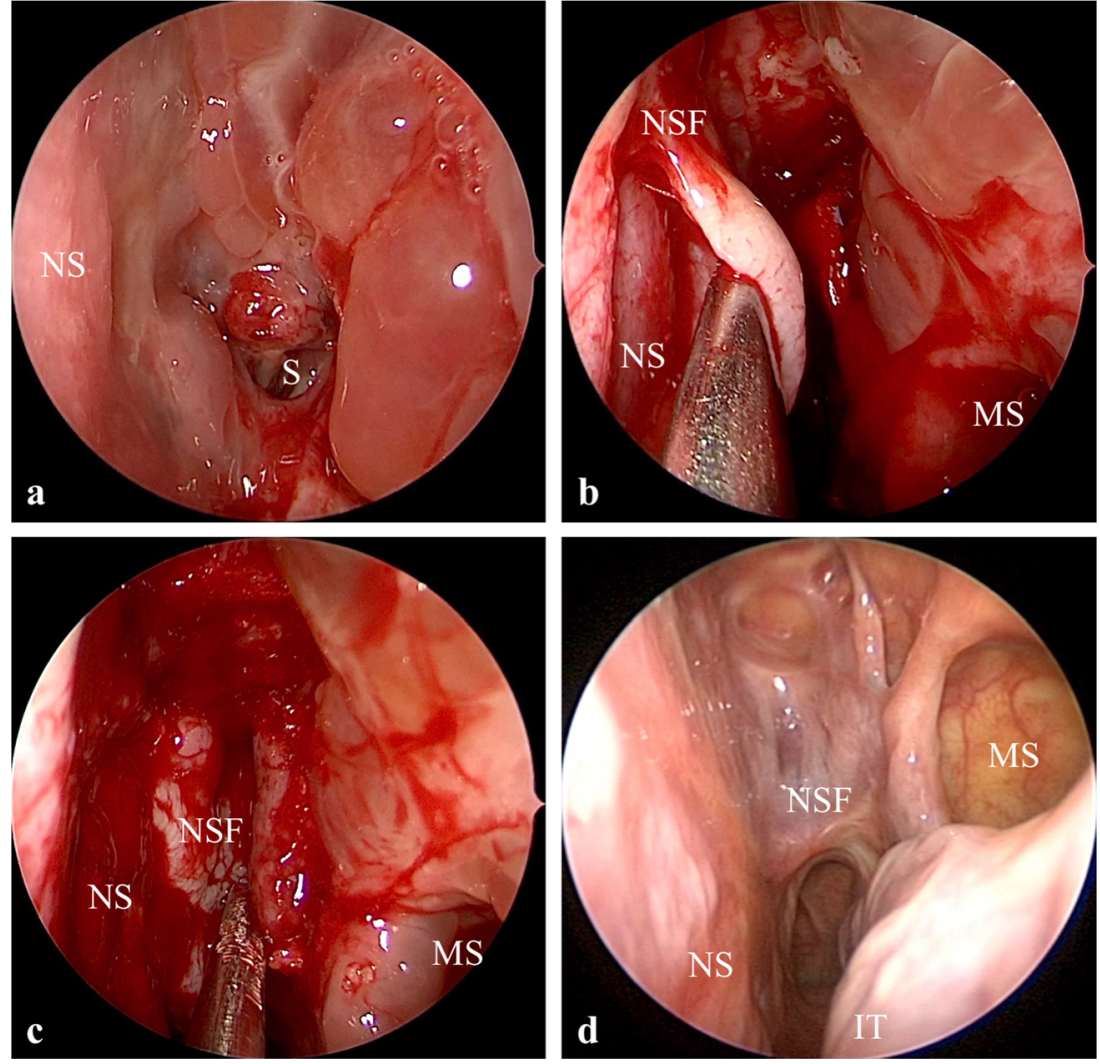

correct positioning, normal patency and flow of both ICA, and the reduced supply of contrast to the pseudoaneurysmatic collection from the vessel lumen. One month after the procedure, a CTA was performed in all patients. A control DSA was performed at 3 and 12 months after the procedure. Figures 2, 3 , and 4 show the most representative images of the preprocedural DSA, the positioning of the FDS, and the postprocedural controls with CTA at 1 month and DSA at 3 and 12 months in some of the treated cases.

When an endoscopic endonasal surgical procedure was performed to reconstruct the extracranial side of the ICA wall ("sandwich technique"), the patients were also followed with seriated endoscopic endonasal medications to maintain the patency of the sphenoid ostium and improve the mucosal healing of the surgical cavity, which usually can be obtained within 2 months after surgery (Fig. 1d).

\section{Results}

We selected 5 patients treated in our center with emergency positioning of FDS on the ICA cavernous tract. Clinical, pathological, and radiological data are summarized in Table 1. Four of the 5 patients were male; the mean age was 47.4 years (range, 21-66 years).
During the DSA, the presence of a PSA of the cavernous ICA was evident in all cases. A carotid BOT [7] was performed during angiographic procedure, resulting in a prominent delay in venous phase of the ipsilateral hemisphere to the occluded ICA in all 5 patients. Furthermore, DSA highlighted the intrinsically curved anatomy of the cavernous ICA at the level of the carotid-ophthalmic passage, making the placement of a covered stent challenging. The endovascular emergency positioning of the FDS obtained a repair of the cavernous ICA tear, with bleeding control and initial slowing of the contrast diffusion in the PSA, in all cases. There was no immediate post-procedural rebleeding in any patient. No thromboembolic cerebral ischemic complications occurred after positioning the stent in any patient.

In two cases, a mild and temporary ICA rebleeding occurred in a period ranging from 5 (case \#4) to 15 days (case \#3), requiring further treatments to strengthen the reconstruction. In such cases, a combined endoscopic endonasal procedure ("sandwich technique") was performed to resurface the outer side of the cavernous ICA, as previously described.

After a mean follow-up of 36.6 months (range, 12-65 months), 4 out 5 patients were alive without evidence of symptoms, without any tardive FDS-related complications, with patency of the intracranial circle at the last control. The remaining patient (case \#5) died due to the intracranial progression of sinonasal cancer. 

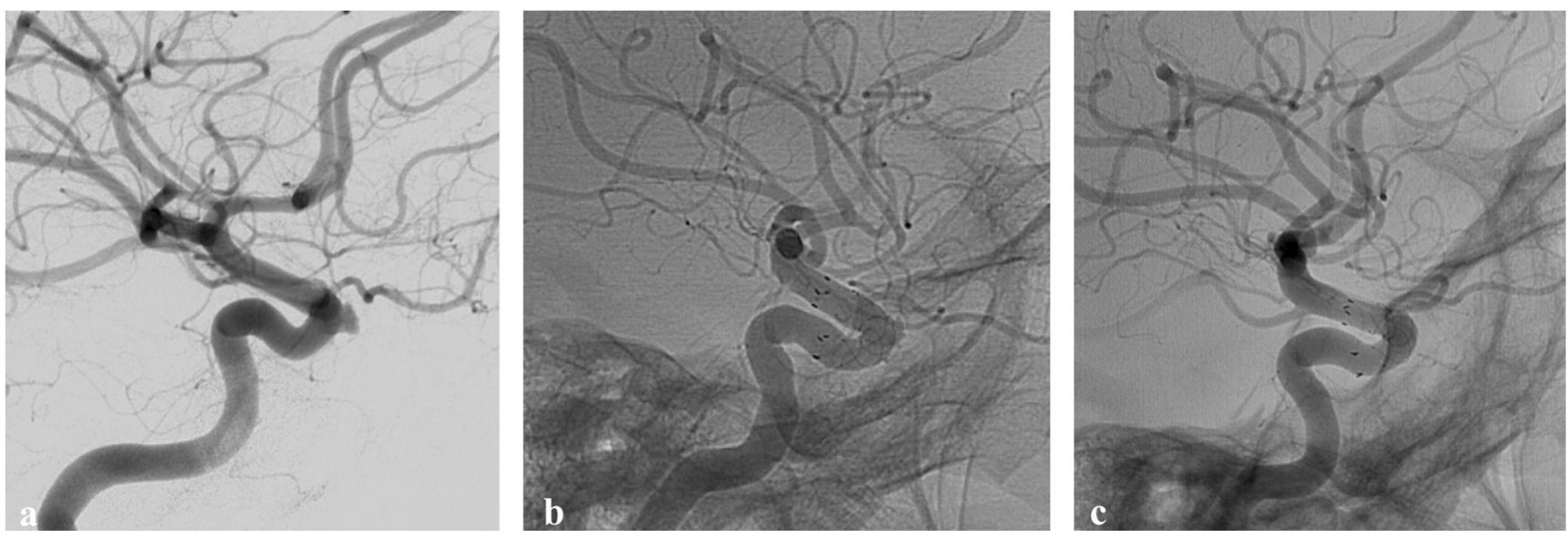

Fig. 2 Case \#3 is a 48-year-old woman admitted to our institution for sudden epistaxis. She was affected by recurrent chronic rhinosinusitis with nasal polyps treated at another center with radical sphenoethmoidectomy with maxillary and frontal sinusotomies about 1 month before. A diagnostic CTA and DSA documented a PSA of the anterior

\section{Discussion}

In this study, we showed our single-center preliminary experience in the management of cavernous ICA acute bleeding, describing for the first time a case series of patients treated in the emergency with endovascular FDS placement, as an isolated procedure or combined with an endoscopic endonasal trans-sphenoidal approach, demonstrating the absence of postprocedural ischemic-hemorrhagic complications in the short and long term.

Managing a cavernous ICA acute injury is a complex issue, as it involves rapid blood loss originating from a challenging to reach anatomical area. There are three options to treat such a condition: ICA sacrifice, ICA lesion embolization, and ICA endoluminal reconstruction $[1-6,9]$.

The ICA sacrifice is the treatment of choice and generally performed after proper verification of the hemodynamic compensation by vascular occlusion test (e.g., BOT). A variable profile of the left carotid siphon. An FDS (FRED, $4 \times 12 / 18 \mathrm{~mm}$ ) was placed. a Pre-procedural DSA showing PSA of the anterior genu right cavernous ICA segment. b Intra-procedural DSA showing FDS correct placement. $\mathbf{c}$ DSA at 3 months, demonstrating the regular cavernous ICA profile, without evidence of PSA recurrence low rate of permanent neurological ischemic deficits after ICA occlusion remains, on average estimated around $0.4 \%$ [7].

In the case of vascular wall lesions with high flow blood loss, the embolization technique represents the best solution, especially when the cavernous ICA acute injury is localized on the lateral wall of the vessel, towards the cavernous sinus. In this case, the blood collects forming a pseudoaneurysmatic pouch, until it breaks generating a carotid-cavernous fistula (CCF), with a direct flow from the carotid artery (higher pressure area) to the cavernous sinus (lower pressure area). The high-speed flow makes CCF treatment by flow diversion challenging given the unfavorable hemodynamics, although some cases of CCF effectively treated with endovascular FDS positioning have been reported in the literature [10-12].

Conversely, when the breach is located on the medial wall of the cavernous ICA, blood collects between the vessel wall and the lateral wall of the sphenoid sinus, forming a PSA. In some cases, the collection increases until the pressure of the
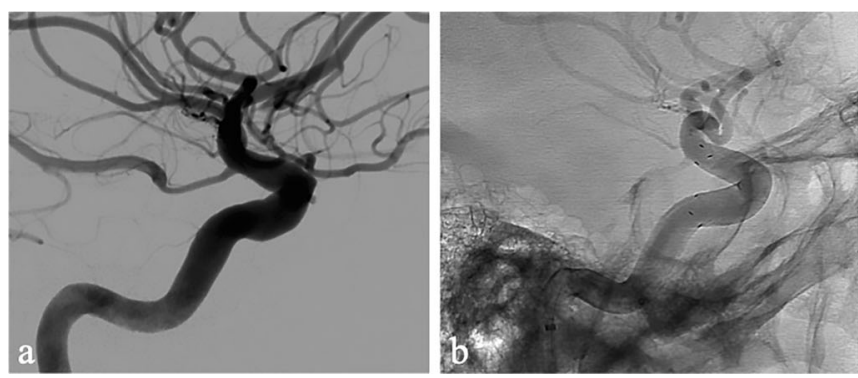

Fig. 3 Case \#4 is a 48-year-old man with pituitary GH-secreting macroadenoma. The lesion was approached with an endoscopic transsphenoidal paraseptal binostril approach. An intraoperative cavernous ICA medial wall damage occurred during the sphenoidotomy. Immediate bleeding control was achieved by direct packing with hemostatic agent (oxidized regenerated cellulose, Surgicel ${ }^{\circledR}$ Original,

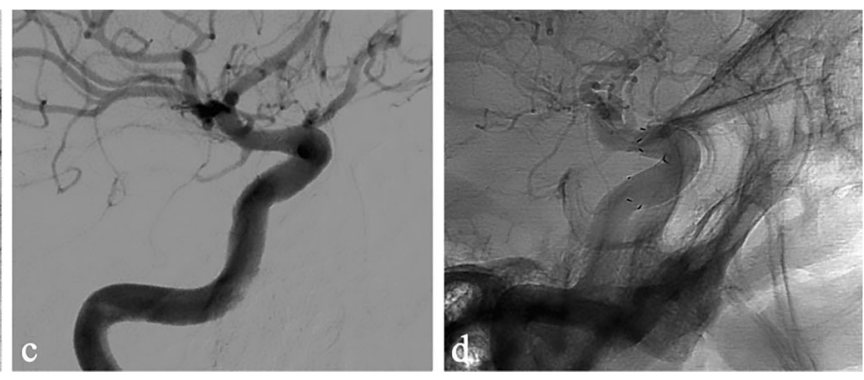

Ethicon, Inc., NJ, USA) of the sphenoid sinus and nasal cavity. The patient was immediately moved to the angiographic room to manage the injured vessel. a Pre-procedural DSA showing a PSA of the anterior genu right cavernous ICA segment. b Intra-procedural DSA showing FDS correct placement. $\mathbf{c}$ and d DSA at 12 months demonstrating the appropriate occlusion of the PSA (c) and correct FDS placement (d) 

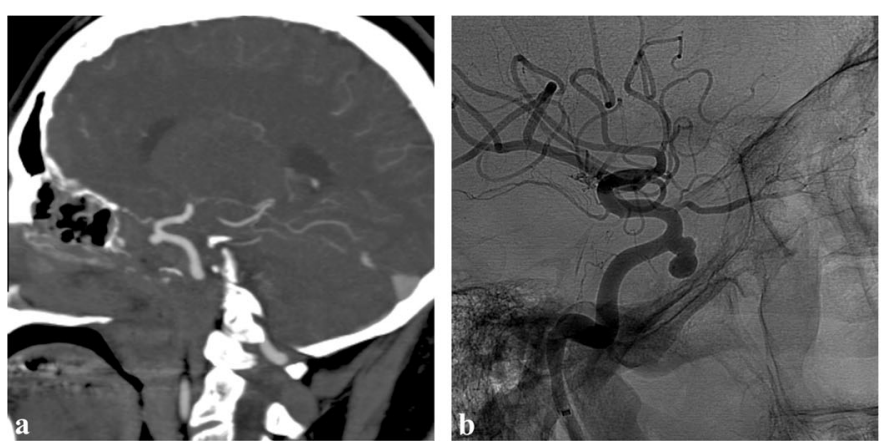

Fig. 4 Case \#5 was a 54-year-old male affected by poorly differentiated sphenoid sinus squamous cell carcinoma (pT4bN0M0), extended bilaterally to cavernous sinuses and with a $360^{\circ}$ encasement of right ICA, previously submitted to chemotherapy ( 2 cycles of cisplatin and paclitaxel) and intensity-modulated proton beam therapy (70 Gy). The patient experienced massive epistaxis, investigated with diagnostic

PSA is such as to damage the thin bone of the sphenoid sinus lateral wall and pour it into the sphenoid cavity. In the case of PSA, the blood flow between the vessel and the pseudoaneurysmatic collection is limited and the FDS can effectively derive the flow and interrupt the discontinuity of the vascular wall.

Over the years, vascular endoluminal reconstruction with stents has emerged as a feasible alternative to occlusion of the damaged vessel. Several cases have been described in the literature where uncovered and covered polytetrafluoroethylene (PTFE) stents were placed to repair arterial tears. Celil et al. [2] and Chen et al. [13] report cases of cavernous ICA PSA treated effectively with covered stent implantation. Ruiz-Juretschke et al. [5] use an overlapping self-expanding uncovered stent for the treatment of posttraumatic cavernous ICA PSA, and Ko et al. [4] describe another case of PSA treatment with a balloon-expandable covered stent. However, these types of stents may have some disadvantages and limitations. The main problem is represented by the reduced flexibility, which limits its applicability to vessels with a tortuous course such as the cavernous and paraclival tract of the ICA. Besides, complications such as stent thrombosis and stent embolism, vascular dissection, malapposition, and endoleak can occur [6, 14-17].

A valid alternative to the uncovered and PTFE stents for the endovascular reconstruction of cavernous ICA tear is represented by the FDS. At present, there are few cases described in the literature of a cavernous ICA acute injury successfully managed with an FDS release. Some cases of iatrogenic cavernous ICA PSA treated in the late post-operative period with FDS are reported in the literature $[1,3,12,18]$. Giorgianni et al. report two cases describing the emergency positioning of FDS for cavernous ICA acute injury, respectively, in a traumatic [3] and iatrogenic cavernous ICA tear [18]. Also Nariai et al. [19] report on a single patient who underwent successful treatment using an FDS for an iatrogenic intracranial internal carotid artery pseudoaneurysm following a cavernous ICA

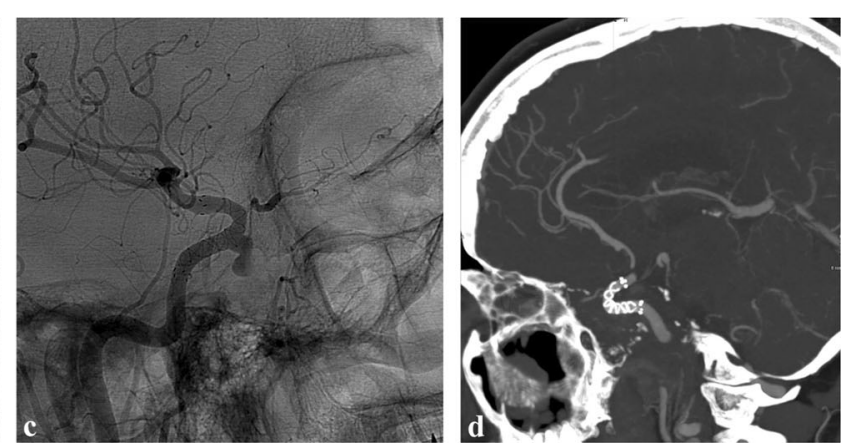

imaging, including CTA and DSA, which showed cavernous ICA PSA. The bleeding was controlled by FDS placement. a and b Pre-procedural CTA (a) and DSA (b) showing anterior genu right ICA cavernous segment PSA. c Intra-procedural DSA showing FDS correct placement. d CTA at 1 month demonstrating PSA resolution

injury after endoscopic pituitary tumor resection via the trans-sphenoidal approach.

Recently, the use of coated FDS for the endovascular treatment of acute ruptured intracranial aneurysms has been described. Among the coated FDS proposed, hydrophilic polymer coating (HPC) $[20,21]$ and pipeline embolization device with shield technology (PED-shield) are currently available $[19,22,23]$. The advantage of coated FDS would be the post-procedural single antiplatelet therapy (SAPT), compared with the double antiplatelet therapy (DAPT) required in noncoated FDS. Early data collected on coated FDS in animal studies and small case series showed less thrombogenicity, faster endothelial growth, and comparable neointimal volume than other non-coated devices [19-23]. However, these are preliminary evidence, with the need for further investigation with a larger number of patients and a formal treatment registry [22]. It must be considered that in our case series, there were no hemorrhagic complications associated with the postprocedural DAPT. Therefore, there is still no demonstrated evidence of the need to use a SAPT in patients with FDS. The use of coated-FDS, as HPC and PED-shield, can instead certainly be useful for patients with hemorrhagic risk factors in whom DAPT is strongly discouraged (e.g., polytraumatized, thrombocytopenic patient).

Furthermore, we showed that the endoscopic endonasal resurfacing of the extracranial wall of the vessel using flaps or grafts can play a synergistic role with FDS endovascular treatment in repairing the vascular wall tear. This combined "sandwich technique" can offer a double support at intra- and extracranial level for the reconstruction of the cavernous ICA wall, reducing the risk of rebleeding. According to our experience, we propose the sandwich technique not as a standard of care in case of FDS placement but as a specific technique to manage rebleeding after FDS placement. This technique has not been yet described in the literature and the outcomes obtained in our two preliminary cases are encouraging, supporting the usefulness of this combined procedure. 
It must be noted that FDS placement has some disadvantages, including the need of post-procedural double antiplatelet therapy, which should be maintained for a few months, and the long time necessary for a complete repair of the vessel $[2,18]$.

The main limitation of this study is represented by a small sample of patients analyzed. Further studies with a larger number of cases and involving multiple centers would be required to validate the results obtained in this study.

\section{Conclusions}

In this study, we have shown the relevant role of the FDS endovascular emergency placement, which can be considered a safe and effective option, alternative to the definitive vascular occlusion, for selected cases of cavernous ICA acute bleeding, regardless of vascular injury caused. The actual indications of this technique are injuries placed in the medial wall of the cavernous ICA, in patients at high risk for neurological sequelae related to the ICA sacrifice, estimated on pre-operative radiological assessments. The combined endoscopic endonasal transsphenoidal resurface of the extracranial intrasphenoidal side of the cavernous ICA, which has been described in the present study for the first time, should be considered in selected cases of rebleeding to further strengthen the vessel wall reconstruction.

Funding Information Open Access funding provided by Università degli Studi dell'Insubria within the CRUI-CARE Agreement.

\section{Compliance with ethical standards}

Conflict of interest The authors declare that they have no conflict of interest.

Ethical approval All procedures performed in studies involving human participants were in accordance with the ethical standards of the institutional and/or national research committee (name of institute/committee) and with the 1964 Helsinki declaration and its later amendments or comparable ethical standards.

For this type of study formal consent is not required.

Additional informed consent was obtained from all individual participants for whom identifying information is included in this article.

Abbreviations ASA, acetylsalicylic acid; BOT, balloon occlusion test; CT, computed tomography; CTA, CT angiography; DAPT, double antiplatelet therapy; DED, Derivo embolization device; DSA, digital subtraction angiography; F, female; FDS, flow diverter stent; FRED, flow-redirection endoluminal device; GCS, Glasgow coma scale; IMPT, intensitymodulated proton therapy; IT, inferior turbinate; iv, intravenous; M, male; MRI, magnetic resonance imaging; MS, maxillary sinus; NS, nasal septum; NSF, nasoseptal flap; PED, pipeline embolization device; PSA, pseudoaneurysm; PTFE, PolyTetraFluoroEthylene; S, left sphenoidotomy; SAPT, single antiplatelet therapy

Open Access This article is licensed under a Creative Commons Attribution 4.0 International License, which permits use, sharing, adaptation, distribution and reproduction in any medium or format, as long as you give appropriate credit to the original author(s) and the source, 
provide a link to the Creative Commons licence, and indicate if changes were made. The images or other third party material in this article are included in the article's Creative Commons licence, unless indicated otherwise in a credit line to the material. If material is not included in the article's Creative Commons licence and your intended use is not permitted by statutory regulation or exceeds the permitted use, you will need to obtain permission directly from the copyright holder. To view a copy of this licence, visit http://creativecommons.org/licenses/by/4.0/.

\section{References}

1. AlQahtani AA, Castelnuovo P, Nicolai P, Prevedello DM, Locatelli D, Carrau RL (2016) Injury of the Internal Carotid Artery During Endoscopic Skull Base Surgery: Prevention and Management Protocol. Otolaryngol Clin N Am 49(1):237-252

2. Celil G, Engin D, Orhan G, Barbaros C, Hakan K, Adil E (2004) Intractable epistaxis related to cavernous carotid artery pseudoaneurysm: Treatment of a case with covered stent. Auris Nasus Larynx 31(3):275-278

3. Giorgianni A, Pellegrino C, Minotto R, Mercuri A, Baruzzi F, Cantoni A, Cardim LN, Valvassori L (2015) Flow-diverter stenting in post-traumatic pseudoaneurysm of cavernous internal carotid artery with epistaxis. Interv Neuroradiol 21(3):325-328

4. Ko JK, Lee TH, Lee JIL, Choi CH (2011) Endovascular treatment using graft-stent for pseudoaneurysm of the cavernous internal carotid artery. J Korean Neurosurg Soc 50(1):48-50

5. Ruiz-Juretschke F, Castro E, Mateo Sierra O, Iza B, Manuel Garbizu J, Fortea F, Villoria F (2009) Massive epistaxis resulting from an intracavernous internal carotid artery traumatic pseudoaneurysm: Complete resolution with overlapping uncovered stents. Acta Neurochir 151(12):1681-1684

6. Sylvester PT, Moran CJ, Derdeyn CP et al (2016) Endovascular management of internal carotid artery injuries secondary to endonasal surgery: Case series and review of the literature. J Neurosurg 125(5):1256-1276

7. Abud DG, Spelle L, Piotin M, Mounayer C, Vanzin JR, Moret J (2005) Venous phase timing during balloon test occlusion as a criterion for permanent internal carotid artery sacrifice. Am J Neuroradiol 26(10):2602-2609

8. Paige ML, Mikhael MA (1991) Intracavernous carotid artery aneurysm presenting as recurrent epistaxis. Ann Otol Rhinol Laryngol 100(7):577-579

9. Bechan RS, Majoie CB, Sprengers ME, Peluso JP, Sluzewski M, Van Rooij WJ (2016) Therapeutic internal carotid artery occlusion for large and giant aneurysms: A single center cohort of 146. Am J Neuroradiol 37(1):125-129

10. Hüseyinoglu Z, Oppong MD, Griffin AS, Hauck E (2019) Treatment of direct carotid-cavernous fistulas with flow diversion - does it work? Interv Neuroradiol 25(2):135-138

11. Iancu D, Lum C, Ahmed ME, Glikstein R, Dos Santos MP, Lesiuk H, Labib M, Kassam AB (2015) Flow diversion in the treatment of carotid injury and carotid-cavernous fistula after transsphenoidal surgery. Interv Neuroradiol 21(3):346-350
12. Wendl CM, Henkes H, Martinez Moreno R, Ganslandt O, Bäzner H, Aguilar Pérez M (2017) Direct carotid cavernous sinus fistulae: vessel reconstruction using flow-diverting implants. Clin Neuroradiol 27(4):493-501

13. Chen D, Concus AP, Van Halbach V, Cheung SW (1998) Epistaxis originating from traumatic pseudoaneurysm of the internal carotid artery: Diagnosis and endovascular therapy. Laryngoscope 108(3): 326-331

14. Chalouhi N, Daou B, Kung D et al (2015) Fate of the Ophthalmic Artery after Treatment with the Pipeline Embolization Device. Neurosurgery 77(4):581-584

15. Dumont A, Starke R, Jabbour P, Tjoumakaris S, Gonzalez L, Rosenwasser R, Pribitkin E, Amenta P (2012) Successful treatment of a traumatic carotid pseudoaneurysm with the Pipeline stent: Case report and review of the literature. Surg Neurol Int 3:160

16. Lehmann P, Saliou G, Page C, Balut A, Le Gars D, Vallée JN (2009) Epistaxis revealing the rupture of a carotid aneurysm of the cavernous sinus extending into the sphenoid: Treatment using an uncovered stent and coils. Review of literature. Eur Arch OtoRhino-Laryngol 266(5):767-772

17. Nerva JD, Morton RP, Levitt MR, Osbun JW, Ferreira MJ, Ghodke BV, Kim LJ (2015) Pipeline Embolization Device as primary treatment for blister aneurysms and iatrogenic pseudoaneurysms of the internal carotid artery. J Neurointerv Surg 7(3):210-216

18. Giorgianni A, Pozzi F, Pellegrino C, Padovan S, Karligkiotis A, Castelnuovo P, Locatelli D (2019) Emergency placement of a Flow Diverter Stent for an Iatrogenic Internal Carotid Artery Injury During Endoscopic Pituitary Surgery. World Neurosurg 122:376-379

19. Nariai Y, Kawamura Y, Takigawa T, Hyodo A, Suzuki K (2020) Pipeline embolization for an iatrogenic intracranial internal carotid artery pseudoaneurysm after transsphenoidal pituitary tumor surgery: Case report and review of the literature. Interv Neuroradiol 26(1):74-82

20. Aguilar-Perez M, Hellstern V, Al Matter M, Wendl C, Bäzner H, Ganslandt O, Henkes H (2020) The p48 flow modulation device with hydrophilic polymer coating (HPC) for the treatment of acutely ruptured aneurysms: early clinical experience using single antiplatelet therapy. Cardiovasc Intervent Radiol 43(2020):740-748

21. Lenz-Habijan T, Bhogal P, Bannewitz C, Hannes R, Monstadt H, Simgen A, Mühl-Benninghaus R, Reith W, Henkes H (2019) Prospective study to assess the tissue response to HPC-coated p48 flow diverter stents compared to uncoated devices in the rabbit carotid artery model. Eur Radiol Exp 3:47

22. Manning NM, Cheung A, Phillips TJ, Wenderoth JD (2019) Pipeline shield with single antiplatelet therapy in aneurysmal subarachnoid haemorrhage: multicentre experience. J NeuroIntervent Surg 11:694-698

23. Matsuda Y, Jang D-K, Chung J, Wainwright JM, Lopes D (2019) Preliminary outcomes of single antiplatelet therapy for surfacemodified flow diverters in an animal model: analysis of neointimal development and thrombus formation using OCT. J NeuroIntervent Surg 11:74-79

Publisher's note Springer Nature remains neutral with regard to jurisdictional claims in published maps and institutional affiliations. 